\title{
Lineamientos de manejo materno-infantil en el consultorio odontológico en tiempos de COVID-19
}

Asociación Latinoamericana de Odontopediatría. Equipo Interdisciplinario COVID-19.

\section{Introducción}

Ante la situación de pandemia de COVID-19, se ha desarrollado el siguiente documento desde la Asociación Latinoamericana de Odontopediatría ALOP, de forma colaborativa y voluntaria, realizando la curaduría de la información científica disponible hasta la fecha de su elaboración.

El objetivo de este documento es brindar orientación técnica a los Odontopediatras y Odontólogos que realizan tratamiento materno-infantil, específicamente de la gestante y del bebé, que permita la toma de decisiones informadas referentes al despistaje, evaluación pre-atención y atención odontológica apropiada durante la pandemia COVID-19, optimizando la calidad de la misma y minimizando el riesgo de transmisión de coronavirus entre pacientes y el equipo odontológico que realiza el tratamiento.

Debe resaltarse que se parte de las mejores recomendaciones posibles, esta información puede ser cambiante de forma rápida por lo que instamos a los profesionales a realizar seguimiento a las actualizaciones de este documento. Asimismo, quedarán muchas preguntas sin respuestas todavía, debido a que los artículos disponibles noestán siguiendo los protocolos habituales para su realización, unos se basan en muy baja casuística, otros indican falta de constatación o tiempos demasiado rápidos para lograr resultados confiables.

Embarazo en el contexto de la atención odontológica

Cabe recordar que durante el embarazo los factores hormonales, microbianos e inmunológicos tienen influencia definitiva sobre los tejidos gingivales, convirtiéndose en factores de riesgo capaces de desencadenar procesos infecciosos importantes.

Desde el año 1994, hay muchos estudios que evalúan la correlación entre infecciones periodontales y desenlaces obstétricos desfavorables, como parto prematuro, nacimiento de bebés de bajo peso y preclamsia. ${ }^{1}$ Revisiones sistemáticas 
recientes sustentan esa correlación, pero resaltan la escasez de estudios clínicos aleatorizados que nos confirmen la relación de causa y efecto. ${ }^{2}$

A pesar de que el tratamiento periodontal no esté científicamente relacionado a la reducción del riesgo de desenlaces obstétricos desfavorables a nivel general, las poblaciones más susceptibles a esos desenlaces parecen beneficiarse más de la intervención odontológica curativa. ${ }^{3}$ Además, la calidad de vida de la gestante puede sufrir impacto de condiciones dento-gingivales inadecuadas, siendo que hay percepción de la mejora después del tratamiento periodontal. ${ }^{4}$

Por lo expresado anteriormente, es necesario diagnosticar las condiciones bucales de la embarazada, identificar los factores de riesgo para caries, enfermedad periodontales $y$ de otras afecciones bucales.

Los objetivos en la atención odontológica a la gestante son:

- Lograr que alcance y mantenga bajo riesgo bucal instalando hábitos de cuidado oral para que llegue al parto sin complicaciones odontológicas que interfieran el normal desarrollo del embarazo y parto.

- Mantenerla informada y entrenada para el cuidado de la salud bucal de su hijo, con el objeto de orientarlas sobre estímulo a la lactancia materna, chupete (chupón) y biberón, alteraciones asociadas a urgencias en neonatos (anquiloglosia, diente natal, épulis congénito) y prevención de la enfermedad caries. Pensando en las gestantes afectadas por COVID-19, principalmente las que desarrollan síntomas como fiebre o inclusive parto prematuro, es importante orientarlas sobre los potenciales riesgos de hipomineralización en dientes primarios de los futuros bebés. ${ }^{5}$

En una situación extraordinaria como la pandemia de COVID-19, esos criterios deben ser evaluadas en cada caso. Aunque el Odontopediatra esté prestando cuidados urgentes, los consejos preventivos habituales no deben ser ignorados, debiendo ser realizados a distancia por medio de la Teleodontología.

Embarazo en tiempos de pandemia por COVID - 19 ¿qué debe saber el odontopediatra - odontólogo?

Los datos sugieren que la infección por SARS-CoV-2 se observa con mayor frecuencia en pacientes adultos varones con una edad media de entre 34 y 59 años. ${ }^{6}$

El SARS-CoV-2 también tiende a generar complicaciones médicas en mayor medida a las personas con comorbilidades crónicas, como enfermedades cardiovasculares, cerebro vasculares y diabetes, por lo tanto si la mujer embarazada cursa un embarazo con los problemas mencionados estaría en riesgo y requeriría mayores cuidados. ${ }^{7}$

A su vez, se dispone de limitada información acerca de la incidencia de infección por SARS-CoV-2 en embarazadas. Sin embargo, la información disponible sobre enfermedades asociadas con otros coronavirus altamente patógenos (es decir, el síndrome respiratorio agudo severo SARS y el síndrome respiratorio del Medio Oriente MERS) podrían proporcionar cierto conocimiento sobre los efectos de la enfermedad por Coronavirus 2019 durante el embarazo. ${ }^{7}$ 
Datos anteriores sobre SARS y MERS sugieren que los hallazgos clínicos durante el embarazo pueden variar, desde ser asintomática hasta presentar enfermedad severa y muerte. Los síntomas comunes de COVID-19 son fiebre y tos, presentes en más del $80 \%$ de los pacientes hospitalizados. $^{7}$

Según dos informes que describen 18 embarazos con enfermedad por Coronavirus 2019, todos se infectaron en el tercer trimestre y los hallazgos clínicos fueron similares a los de los no embarazados. ${ }^{9}$

Es por ello que a pesar de esta pequeña población analizada, se considera hasta el momento, a la mujer embarazada como un adulto normal. ${ }^{10}$

\section{Relación del COVID-19 con la práctica odontológica en la embarazada}

Durante la etapa de confinamiento se debe realizar tratamiento odontológico a pacientes embarazadas que presenten emergencias y urgencias, como así también en tiempos de no confinamiento, y como el que se realiza en los adultos que no cursen embarazo. También está indicado diferir todo tratamiento odontológico electivo, hasta tanto sea vez resuelta la pandemia y finalizado el embarazo. ${ }^{11,12}$

Ruta de atención para procedimientos de odontología para gestantes durante la etapa de confinamiento o cuarentena de la pandemia COVID-19

Es importante que, no sólo se debe realizar cualquier tipo de atención odontológica durante el embarazo como en cualquier otro paciente-, definida como del tipo urgencia o emergencia, sino que también el profesional que realizará el tratamiento deberá estar actualizado sobre el curso de la pandemia en su localidad, de esta manera se podrá brindar una atención siguiendo las regulaciones del Ministerio de Salud del país donde se encuentra. ${ }^{13}$

\section{Teleodontología y Triage}

Diferentes organismos internacionales, incluyendo la Asociación Latinoamericana de Odontopediatría, recomiendan el uso de Teleodontología como primera línea de atención. ${ }^{14}$

La ADA (Australian Dental Association Asociación Odontológica Australiana) especifica que la consulta por audio o mediante cualquier plataforma de videoconferencia es una herramienta útil, cuando una embarazada que no puede asistir a un consultorio o clínica dental, como es el caso en tiempos de pandemia- confinamiento por COVID-19, requiere atención odontológica urgente. Los profesionales intervinientes deberán asegurarse de que la solución del sistema de telecomunicación elegida cumpla con los requisitos clínicos y satisfaga la privacidad de las consultas. ${ }^{15}$

Sin embargo, cada país y sus consejos de reglamentación de la profesión pueden tener sus normas para la práctica de Teleodontología. En América Latina las reglas difieren para cada país, por eso, a pesar de presentar una posición sugerida, es importante que cada profesional analice como la ley de su país se posiciona. ${ }^{14}$

La consulta inicial telefónica se realizará para la identificación de pacientes 
embarazadas con sospecha o posible infección con COVID -19, de manera remota al momento de programar la cita.16

Las 3 preguntas iniciales estarán vinculadas para definir si se está ante la presencia de una paciente sospechosa o portadora de COVID- 19, presencia de síntomas de enfermedades respiratorias con fiebre o tos. ${ }^{13}$ Es importante destacar que al existir transmisión comunitaria del virus, el tamizaje inicial es importante, sin embargo tiene limitaciones para identificar pacientes infectados asintomáticos o presintomáticos, por lo que todos los pacientes deberían ser tratados como si fueran postencialmente "positivos".

De cualquier forma, ante una respuesta positiva a cualquiera de las 3 preguntas mencionadas, se deberá referir a la paciente para el manejo médico odontológico por COVID-19 y trabajar en conjunto con el equipo médico para resolver la urgencia o emergencia odontológica. No tratar o no medicar una emergencia y urgencia odontológica en un mujer embarazada puede poner en riesgo el desarrollo normal del parto. Como se dijo anteriormente, se le proporciona en esta instancia toda la información sobre medidas de auto cuidado personal y cuidados para su bebé. ${ }^{16,17}$

Considerando que las gestantes son pacientes especiales, en muchos casos portadoras de patologías asociadas, las enfermedades dentales y gingivales podrían potencializar los riesgos para desenlaces desfavorables. Siendo así, no es adecuado postergar la atención de urgencia/emergencia de la gestante.

Cabe decir, que en el caso de una mujer embarazada, el profesional deberá favorecer el uso de una teleconsulta sincrónica, en tiempo real, para actuar con rapidez y continuar con la definición de la ruta de tratamiento, el lugar de realizar una primera consulta asincrónica, donde hay un tiempo de espera para recibir la evaluación. ${ }^{18}$

Adicionalmente al cuestionario previo, se debe incluir una guía explicita acerca de las características de consulta virtual que debe ser firmada bajo el acuerdo que, al realizar la consulta, se aceptan las estipulaciones allí contenidas, incluyendo especificaciones sobre el alcance, las limitaciones y los pasos básicos a seguir ya sea de forma sincrónica o asincrónica.

\section{Anamnesis y definición del motivo de consulta}

Con la atención siendo realizada a distancia, se considera que el diagnóstico puede ser presuntivo y no definitivo. Por esoesimportante quela consulta a distancia sirva como un triage, direccionando a la gestante para la atención presencial de ser necesaria.

Para la gestante con diagnóstico negativo COVID-19, de ser necesaria la consulta presencial, debe determinarse o seguir los pasos apropiados para brindar la atención óptima, guardando todas las medidas de bioseguridad.

En caso de emparazadas con diagnóstico de COVID-19, será referida para manejo médico correspondiente y se realizará la atención en conjunto con el equipo interdisciplinario.

En Odontología se ha establecido que 
los motivos más frecuentes de consulta por urgencia son: dolor, inflamación, traumatismo o hemorragia. Es necesario indagar información referente a estos síntomas, utilizando criterios de gravedad, para poder brindar orientación $\mathrm{y}$ tratamiento al paciente.

En todos los casos, se debe mantener el seguimiento de su evolución, utilizando las mismas vías de comunicación. ${ }^{19}$

\section{Registro físico de la consulta virtual sincrónica}

Al realizar atención por Teleodontología es indispensable llevar un registro de los datos con una ficha diseñada que incluya previamente toda la información referente a la historia médica que se tenga del paciente, o datos referidos por otro especialista. Igualmente se deberá registrar todo lo relativo al episodio, que sirva al clínico como guía al momento de realizar un diagnóstico presuntivo y que le permita mantener el seguimiento por escrito de la teleconsulta o consulta virtual. Anexo a este artículo se propone un modelo de registro durante la consulta virtual sincrónica en Odontología para la gestante. ALOP ha publicado previamente guías para la teleodontología dirigidas la paciente pediátrico. ${ }^{14}$

De esta manera, cuando el especialista sea contactado por el paciente para solicitar una consulta virtual, deberá abrir un nuevo registro que apoyará su gestión clínica y todos los respaldos legales de su práctica profesional.

Este registro deberá contener información básica como fecha de contacto, iniciales razones de consulta, nombre de la persona que inicio el contacto, nombre del paciente, si es paciente nuevo o parte de la consulta de su clínica, entre otros.
Este registro inicial de requerimiento de consulta puede ser llenado virtualmente y almacenado digitalmente, mientras que las condiciones profesionales, permitan incluir este documento dentro de la Historia Clínica del paciente ubicada en la clínica o consultorio.

\section{La consulta presencial}

Se sugiere que la consulta presencial sea realizada principalmente en los casos de urgencia descritos anteriormente. Para esto es importante que el profesional siga rigorosamente las medidas de bioseguridad, así como aquellas adoptadas para la atención infantil. ${ }^{19}$

En gestantes se sugiere que la atención clínica presencial sea realizada para el adecuado diagnóstico y tratamiento clínico de las siguientes condiciones:

- Absceso dento-alveolar agudo con o sin señales de diseminación

- Pulpitis irreversible

- Pulpitis reversible con dolor provocado

- Trauma dental o facial

- Sangrado gingival exacerbado

- Otras condiciones que incomoden a la gestante y merezcan atención presencial para que no haya interferencia en su calidad de vida.

\section{Orientaciones inmediatas y tardías para la gestante después de la resolución de la urgencia}

Después de la atención clínica, la gestante merece recibir el seguimiento de la resolución de la urgencia y de las orientaciones preventivas brindadas, En época de pandemia, se sugiere que esa 
consulta se haga a distancia con el uso de Teleodontología.

\section{Recomendaciones sobre la salud bucal del bebé}

Regularmente se aconseja a las mujeres embarazadas que realicen el prenatal odontológico en dos etapas. En la primera, hay atención y orientación sobre salud bucal en una mujer embarazada. En la segunda, la consulta está destinada a una conversación con una mujer embarazada y su acompañante para que comprendan todos los cuidados que deben tener cuando nazca el bebé. ${ }^{20}$ Estas directrices han sido investigadas y respaldadas por importantes revisiones sistemáticas, mostrando el potencial para la prevención, incluida la enfermedad de caries. ${ }^{20,21}$

Durante este período de pandemia, se sugiere que se siganlas mismas pautas, pero por Teleconsulta. En este caso, se puede afirmar que no se perjudica la atención ni es neceario hacer una gran adaptación, ya que la consulta sería meramente informativa. Además, la posibilidad potencial de prevenir enfermedades dentales en futuros bebés, con el enfoque hacia la mujer embarazada, hace que este contacto virtual sea tan importante como el destinado al tratamiento de la mujer embarazada.

\section{Lactancia materna y COVID-19}

La leche materna contiene todos los nutrientes, los componentes antiinflamatorios y los factores inmunológicos -en proporciones idealespara el crecimiento y protección de los bebés, quienes se benefician de la lactancia inclusive en casos de madres con sospecha o confirmación de COVID-19. ${ }^{22}$

El siguiente trabajo tiene como objetivo responder a las preguntas más importantes en relación a la lactancia materna y a la enfermedad por el nuevo coronavirus.

¿Qué dice la evidencia científica sobre el contagio por COVID-19 a un bebé a través de la leche materna?

Hoy por hoy, no queda claro si es posible transmitir el coronavirus verticalmente a través de la leche materna, ya que las muestras de leche materna recolectadas de mamás con COVID-19, dan negativas para SARS-CoV-2. ${ }^{22-24}$ Por lo tanto, parece poco probable que la enfermedad pueda transmitirse al amamantar o a través de la leche materna extraída de una mujer considerada caso sospechoso o confirmado de COVID-19. Hay expertos que inclusive afirman que la leche materna de una mamá infectada contiene anticuerpos específicos para SARS-CoV-2, lo cual modula la expresión clínica de la enfermedad en su bebé. $^{25}$

Lo que parece más probable, es que una madre infectada pueda transmitir el coronavirus a su bebé a través de transmisión horizontal por medio de gotitas respiratorias durante la lactancia o al estar en íntimo contacto sin equipo de protección individual. ${ }^{26,27}$ Las revisiones sistemáticas ${ }^{24,28}$ confirman que a pesar de que existen casos confirmados de neonatos positivos por COVID-19, la infección es muy infrecuente y usualmente asintomática. Asimismo, la última revisión sistemática ${ }^{28}$ constata que no hay mayor riesgo de contagio independiente del tipo de parto (vaginal o cesárea), tipo de alimentación infantil (lactancia materna o fórmula), ni con el contacto cercano con la madre (separación o contacto íntimo). 
¿Cuáles son las recomendaciones respecto a la lactancia materna en tiempos de pandemia?

Ante la falta de evidencia científica de la transmisión de COVID-19 vía la leche materna y como la lactancia materna reduce el riesgo de infecciones por otros patógenos que causan enfermedades graves (especialmente infecciones respiratorias similares al coronavirus), las principales instituciones científicas y públicas $^{29-38}$, apoyan el iniciar y mantener la lactancia materna en bebés de madres infectadas por COVID-19.

Una madre con coronavirus debe recibir apoyo inmediato de los profesionales de la salud para amamantar de forma segura, mantener a su bebé piel con piel y respetar el alojamiento conjunto madrebebé. Asimismo, una madre que adquiere COVID-19, debe seguir amamantando ya que la lactancia materna reduce la mortalidad en neonatos, lactantes y niños menores de 5 años, incluso en entornos con recursos altos. ${ }^{32}$

¿Cuáles son las precauciones que debe tener una madre positiva por COVID-19 al amamantar a su bebé? (Ver Tabla 1)

Según la OMS y otras academias científicas, ${ }^{25,32-34,38}$ al momento de amamantar a sus bebés, las mamás COVID-19 positivas deben:

o Estar aisladas en sus habitaciones ( $\sin$ recibir visitas externas al núcleo familiar)

o Utilizar una mascarilla quirúrgica que cubra su boca y nariz

o Lavarse las manos con agua y jabón 20 segundos antes y después de tocar a su bebé

o Limpiar y desinfectar las superficies que han tocado de manera rutinaria.
¿Qué debe hacer una mamá que se encuentra demasiado indispuesta por coronavirus como para amamantar a su bebé?

Las alternativas propuestas son $25,32,33,38$ :

o Extracción de leche y conservación (sin pasteurizar)

o Recibir donaciones de leche materna de bancos de leche

o Debe estudiarse la compatibilidad de la lactancia materna con toda aquella medicación que reciba la madre infectada. Generalmente los medicamentos actualmente utilizados en COVID-19 muestran compatibilidad con la lactancia materna, aunque siempre deben ser verificados.

o Relactación

Manejo clínico del recién nacido en tiempos COVID-19

\section{Anquiloglosia}

El tratamiento de la anquiloglosia no está incluido en la lista de tratamientos de urgencia. Sin embargo hay diferentes aspectos a tomar en cuenta ya que puede considerarse una situación emergente o urgente.

En caso de que la anquiloglosia del bebé esté afectando la lactancia materna ¿cuál debe ser el manejo del Odontopediatra en tiempos de COVID-19?

La frenotomía o frenectomía lingual no está incluida en la lista de tratamientos de urgencia o emergencia dental durante la pandemia por las diferentes academias odontológicas. ${ }^{39-42}$ Sin embargo, dentro de los procedimientos odontológicos durante la pandemia, la frenotomía o frenectomía estaría considerada dentro 
Tabla 1: Recomendaciones de seguridad para madres y profesionales de salud en las maternidades y servicios de neonatología 22

\section{Recomendaciones para madres que amamantan}

- Higiene respiratoria, inclusive al amamantar (cubrirse la boca y la nariz con el codo al toser o estornudar, o usar papel que se descarte rápidamente).

- En caso de síntomas respiratorios (ej. respiración entrecortada): usar una mascarilla quirúrgica al estar cerca al bebé amamantado.

- Lavarse las manos profusamente con jabón o gel por al menos 20 segundos, antes y después de tocar al bebé.

- Limpiar y desinfectar constantemente las superficies que han sido tocadas.

- Si se encuentra muy enferma con COVID-19, si se sospecha de tener COVID-19 o si existen otras complicaciones que son un obstáculo para continuar con la lactancia directa, extraerse leche para ofrecerla al bebé.

- Si las condiciones clínicas impiden la lactancia o la extracción de leche materna, evaluar la posibilidad de iniciar una relactación (reiniciar la lactancia luego de un periodo de tiempo), o utilizar leche materna donada a través de un banco de leche.

Recomendaciones para profesionales de la salud

- Ningún profesional de la salud trabajando en una maternidad o un servicio de neonatología, debe promover los sustitutos de la leche materna (fórmula), biberones, tetinas o chupones.

- Se les debe permitir a las madres y sus bebés: permanecer en alojamiento conjunto día y noche y practicar el contacto piel con piel; independientemente de si el bebé tiene COVID-19 o si se sospecha de la enfermedad. Este punto es especialmente importante inmediatamente después del nacimiento, cuando se está estableciendo la lactancia materna.

las intervenciones quirúrgicas de riesgo reducido, al no producir aerosoles. ${ }^{43}$

Tomando en cuenta los enormes beneficios de la lactancia materna en épocas de pandemia y que son muy raros los eventos adversos reportados con la frenotomía, siempre que la anatomía del frenillo esté impidiendo la correcta lactancia y las indicaciones estrictas para este procedimiento sean consideradas, esta intervención debe puede constituir una urgencia neonatal. Todo Odontopediatra o Cirujano Maxilofacial capacitado para hacer este procedimiento, debe considerarlo como un tratamiento prioritario, con el fin de apoyar la lactancia materna.

\section{Dientes natales y COVID-19}

El tratamiento del diente natal no ha sido incluido en la lista de urgencias/ emergencias dentales durante la pandemia de COVID-19 por las diferentes academias odontológicas. ${ }^{39,40}$

\section{¿Cuáles son las repercusiones del diente natal en tiempos de COVID-19?}

La presencia de esta patología, en algunos casos, puede generar úlceras en la lengua del bebé (Ulcera de Riga - Fede) ${ }^{44}$ y también lesiones en el pezón de la madre lo que podría generar un desmame precoz, ambos eventos indican la necesidad de intervención y pueden ser considerados como urgencias neonatales, porque 
pueden afectar la lactancia materna, lo que implica la privación sus enormes beneficios en épocas de pandemia.

Otro de las consideraciones por los cuales los dientes natales y neonatales constituyen urgencia odontológica es por la extrema movilidad de los mismos que pueden provocar riesgo de aspiración por el bebé. ${ }^{45}$

\section{¿Cuándo debe realizarse una intervención del diente natal en tiempos de COVID-19?}

En casos cuando no hubiera incomodidad por parte del bebé y la madre, solo se deberán realizar controles, postergando la toma radiográfica para determinar la naturaleza de la pieza dentaria: supernumeraria o de la serie normal. Esto debido a que las radiografías intraorales pueden estimular el flujo salival y generar tos, aumentando el riesgo de infección por COVID-19. ${ }^{46} \mathrm{Si}$ el diente se encuentra fijo o con leve movilidad y presenta un borde rugoso, se realizará en estos tiempos el pulido manual de la esta zona siempre con ayuda de lijas.

En los casos en los cuales se vea comprometida la lactancia, haya presencia de úlceras extensas en la cara ventral de la lengua o haya movilidad extrema de los dientes que constituya riesgo de aspiración, se puede indicar la extracción. Este procedimiento puede ser considerado de bajo riesgo por ser rápido, no generar aerosoles, aunque siempre y cuando el odontopediatra esté capacitado para realizarlo.

\section{Manejo clínico para la primera infancia en tiempos COVID-19}

Según la Organización Mundial de la
Salud, en conformidad con los criterios internacionales para la formulación de políticas, la primera infancia se define como la etapa comprendida desde el desarrollo prenatal hasta los ocho años de edad. ${ }^{47}$ De acuerdo con los descriptores en Salud (DeCS) el recién nacido es el bebé entre durante los 28 días después del nacimiento, infante es el niño entre 1 a 23 meses de edad, y preescolar el niño entre 2 a 5 años de edad.

\section{Transmisión vertical de SARS-CoV-2}

Revisiones sistemáticas ${ }^{48,49}$ describen que aún no existe evidencia científica acerca de la transmisión vertical del SARSCoV-2, así como evidencia muy limitada con relación a la presencia del virus en la leche materna. ${ }^{49,50}$

En recién nacidos y niños el SARS-CoV-2 afecta con menos frecuencia y gravedad a los niños. ${ }^{49}$ El 95\% de todos los casos en niños varían entre casos asintomáticos o con signos de infección principalmente leves (42\%) y moderados (39\%). La presentación clínica en recién nacidos podría ser ligeramente diferente a la de los niños mayores. ${ }^{49}$

En resumen, la mejor evidencia hasta la fecha ha demostrado que los pacientes pediátricos con COVID-19, tuvieron un buen pronóstico y se recuperaron dentro de 1 a 2 semanas después del inicio de la enfermedad. ${ }^{51}$

\section{Uso de mascarillas, chupón y CO V ID-19}

Con relación al uso de mascarillas, la Sociedad Brasileña de Pediatría (SBP), ${ }^{52}$ la Academia Americana de Pediatría $(\mathrm{AAP})^{53}$ y el Centro para el Control y la Prevención de Enfermedades de Estados Unidos $(\mathrm{CDC})^{54}$ desaconseja que los 
niños menores de 2 años usen mascarillas debido al riesgo de asfixia. Por otro lado, discutir sobre el uso del chupón por bebés en tiempos de pandemia es especialmente importante. De acuerdo con la SBP, los niños que usan chupón (chupete) no deberían usar mascarillas, debido al peligro del uso conjunto para mayor riesgo de asfixia. La SBP destaca que los chupones pueden aumentar el riesgo de infección y por ello, requieren no sólo de una cuidadosa higiene, sino también de mucha precaución en su uso.

\section{Riesgo de infección del chupón (chupete, mamadera) y COVID-19}

En el contexto de COVID-19, el chupón podría considerarse un elemento de riesgo de infección, ya que está en íntimo contacto con la boca y el rostro del niño. Los chupones están en contacto con la saliva y la microbiota oral, lo que conduce al desarrollo de biopelículas microbianas en sus superficies. ${ }^{55,56}$ Las caídas de los chupones son frecuentes, lo que expone a los bebés a una amplia gama de microorganismos, y a menudo se devuelve a la boca sin limpieza previa. ${ }^{55}$ Si los chupones se consideran reservorios potenciales de infección, podrían afectar el sistema inmunológico del niño, ${ }^{57}$ más aún en tiempos de COVID-19. Su uso se asocia con una mayor incidencia de enfermedad diarreica y con una mayor probabilidad de hospitalización y eventos de sibilancias, asma, dolor de oído, vómitos, fiebre, diarrea, cólico, aftas y candidiasis oral. ${ }^{56,58}$

Ante esta situación, la desinfección adecuada de los chupones se vuelve esencial, considerando su potencial de infección y transmisión demicroorganismos, las mayores tasas de complicaciones graves por COVID-19 observadas en bebés menores en comparación a los niños mayores, la inmadurez de su sistema inmune y la recomendación clara de no usar mascarillas en menores de 2 años.

\section{¿Cuáles son las recomendaciones con relación al uso de chupones en tiempos de pandemia?}

Una revisión sistemática basada en las directrices del PRISMA, evaluó la mejor estrategia de método de desinfección de chupones en el contexto de COVID-19. ${ }^{59}$ Los autores concluyeron que debido a los diversos métodos de desinfección de chupones y a la accesibilidad a diferentes agentes desinfectantes, el consenso sobre la descontaminación del chupón sigue siendo incierto. Sin embargo, los métodos que mostraron mejores resultados de desinfección de algunos microorganismos tales como Cándida Albicans incluyeron el spray de Digluconato de Clorhexidina al 0,12\% y la desinfección con agua hervida durante 15 minutos. Asimismo, el microondas en el nivel de potencia 7 (70\% de la potencia total) durante 7 minutos y a plena potencia durante 5 minutos, también fueron eficaces en la desinfección del chupón. Sin embargo, este procedimiento debe evaluarse con precaución debido a los diferentes parámetros (niveles de potencia y modelos) de los modelos de microondas.

Por otro lado, en el contexto de pandemia se podría sugerir a las madres optar por chupones con tetinas de silicona, ya que aparentemente las tetinas de silicona, comparadas con las de látex, tienen menor potencial de contaminación por su menor adhesión microbiana. ${ }^{56}$ Cabe mencionar que los chupones de silicona también tienen potencial para la adhesión de microorganismos y podrían ser una fuente de infección. ${ }^{55,56}$

En resumen, debido a los potenciales riesgos de infección por el uso del 
chupón en tiempos de COVID-19, desaconsejaríamos su uso en bebés y niños. No obstante, si el uso fuera una decisión del núcleo familiar, se podría optar por el uso de tetinas de silicona y recomendar una óptima desinfección diaria de los mismos.

\section{Procedimientos odontológicos específicos} en para la primera infancia en tiempos de COVID-19

La OMS ha descrito que una pandemia tiene seis fases diferentes, ${ }^{60}$ y por ello, los países estarán en diferentes fases en diferentes momentos. Este hecho implica que no es posible dar pautas universales sobre cuándo y cómo iniciar las atenciones clínicas presenciales, siendo esencial seguir las pautas actualizadas de cada país para el ejercicio odontológico. ${ }^{61}$

\section{La Ruta de Atención para Procedimientos} de Odontología Pediátrica Durante el Confinamiento o Cuarentena Pandemia de COVID-19 es la herramienta que ALOP ha puesto a disposición de los Odontopedíatras para la atención de Urgencias las Emergencias deberán ser atendidas en centros preparados. ${ }^{19}$

De igual manera, a pesar de que el uso de la Teleodontología representa una herramienta útil en tiempos de pandemia, ésta debe seguir las resoluciones de los órganos responsables de cada país, en cada fase de la pandemia. Por ejemplo, en algunos países tales como Brasil, está expresamente prohibido ejercer la Teleodontología con fines de consulta, diagnóstico, prescripción y preparación de un plan de tratamiento odontológico. ${ }^{14,62}$ En otros países como el Perú, por ejemplo, la Teleodontología no está regulada aún a nivel institucional.
¿Cuáles son las medidas de bioseguridad a tomar en cuenta?

Las precauciones universales de bioseguridad deben seguirse rutinariamente y a rigor para la atención clínica odontológica durante todas las fases de la pandemia. Estas son fundamentales para evitar la transmisión del virus SARSCoV-2 a los niños, así como la transmisión de niños infectados a profesionales de la salud. ${ }^{61}$

Las precauciones universales de bioseguridad pueden ser revisadas en la Ruta de atención para procedimientos de Odontología Pediátrica durante la etapa de confinamiento o cuarentena de la pandemia COVID-19 publicados por la ALOP. ${ }^{19}$

¿Existen medidas
en el tratamiento
odontopediátrico?

Resulta importante destacar que, durante la pandemia por COVID-19, no está indicado el uso del manejo de conducta farmacológica en la atención odontológica, debido a mayores riesgos de contaminación. ${ }^{63,64}$

Las consideraciones son las siguientes:

Debido a la aerosolización generada por el flujo de gases con el óxido nitroso, al mayor riesgo de propagación del virus $\mathrm{y}$ al potencial riesgo de realizar medidas de soporte básico de vida que involucren el manejo de las vías respiratorias y el uso de recursos hospitalarios, ${ }^{63,64}$ los procedimientos dentales bajo sedación ambulatoria o el uso de óxido nitroso NO están indicados durante el tiempo de pandemia por COVID-19. ${ }^{63}$ Recordando que el uso de sedación odontológica en bebés menores de 2 años de edad no sería recomendado debido a la falta de respuesta verbal, proceso de madurez 
orgánica aún no concluido, estructuras anatómicas en desarrollo y alto riesgo de evento adverso. ${ }^{65,66}$

Asimismo, cabe recordar que, durante la pandemia, existen restricciones para la atención dental bajo anestesia general debido a la alta tasa de ocupación de camas hospitalarias utilizadas para la atención de pacientes con coronavirus y debido al riesgo de contaminación durante la hospitalización para realizar anestesia general. ${ }^{63,64}$ Los procedimientos bajo anestesia general pueden también generar aerosoles, como en la intubación orotraqueal, ventilación mecánica no invasiva y suministro de oxígeno de alto flujo. ${ }^{64}$ Por ello, la atención clínica odontológica bajo anestesia general para bebés y niños sólo estaría indicada en casos graves, en ambientes clínicos especialmente diseñados y equipados, utilizando técnicas resolutivas para resolver definitivamente el problema dental, evitando tratamientos adicionales a corto plazo. ${ }^{63}$

A partir de lo expuesto, para la atención clínica odontológica y manejo del comportamiento de bebés y niños, sea por motivos de urgencia/emergencia en la fase aguda de la pandemia por la COVID-19, o sea para procedimientos odontológicos electivos en el periodo posterior al pico, se recomienda el uso de técnicas no farmacológicas para controlar la ansiedad del paciente, por ejemplo, aquellas asociadas o no con la estabilización protectora (bebés y personas con discapacidad), y uso técnicas de odontología de mínima intervención. De acuerdo con la $\mathrm{OMS}^{47}$ el período posterior al pico significa que la actividad pandémica parece estar disminuyendo; sin embargo, no está claro si ocurrirán olas adicionales y los países deberán estar preparados para una segunda ola.
Así, el abordaje del comportamiento no farmacológico del bebé es la opción de atención clínica más recomendada en los tiempos de pandemia por la COVID-19, y que se puede trabajar en la fase post-pandemia a criterio clínico del Odontopediatra y respetando las preferencias y decisión del núcleo familiar. Para el uso de estabilización física protectora es necesario Consentimiento Informado y aclarado firmado para el uso ya en la primera consulta. ${ }^{67}$ Durante la fase aguda y periodo posterior al pico de la pandemia por la COVID-19, debe darse preferencia a la estabilización física protectora activa realizada por el propio representante sin el uso de dispositivo adicional para reducir riesgo de contaminación.

\section{Teleodontologia}

Siguiendo los lineamientos de la ALOP, ${ }^{14}$ se realiza la adaptación del uso de teleodontología para ser aplicada en niños entre 0 y 4 años de edad.

La situación de urgencia puede manejarse con Teleodontología. Como establece la Ruta ALOP, ante el requerimiento de Teleconsulta el profesional deberá establecer dos caminos a seguir:

Consulta telefónica o por medios audio-visuales será el modo de contacto primario entre Odontopedíatra y la familia, en orden de evitar la circulación del niño en su traslado hacia la consulta y su exposición en el lugar, será el modo de elección en aquellos países en los que los movimientos del niño estén restringidos.

Atención presencial. Solo cuando la anterior hubiese fracasado o no fuera suficiente por dolor o traumatismo que requiera intervención. 


\section{Teleconsulta:}

$\mathbf{1}^{\circ}$ Llamada o contacto del familiar buscando al Odontopedíatra. Se obtendrán: Datos del niño y de su familia. (Nombres, composición), número de celular o correo electrónico de contacto. Motivo de la consulta, escuchando atentamente al que llama. Las preguntas deben orientar hacia el despistaje de dolor (Cuánto hace? espontáneo ? al estímulo? despertó de noche el dolor? hay edema? ¿Está recibiendo alguna medicación? ¿Quien la indicó?), traumatismos u otro motivo urgente. Es importante establecer si existe relación previa del paciente en la consulta y si ha tenido experiencias anteriores.

Es recomendable intentar interactuar directamente con el miño, sin insistir ni obligar, por lo que se puede solicitar al representante que realice la llamada o videoconferencia con el niño cerca ( $i$ Está el niño contigo ahora? Acércalo al teléfono. Me gustaría hablar con él.)

Indagar posibilidad de exposición o contagio con COVID-19 Preguntar a los padres si ¿tuvo la enfermedad? ¿tiene fiebre, u otros síntomas de COVID-19? ¿hay historia de referentes adultos con estos síntomas dentro y fuera del hogar?

Solicitar al representante que apoye la consulta con imágenes que puedan ayudar a la orientación diagnóstica, que tome una foto o un corto video de la lesión o motivo por el cual consulta.

Incluir en la información todo lo referente a la rutina de higiene habitual, pueden mostrar los cepillos de dientes en sus manos y la pasta dental que usan.

Los datos pueden obtenerse de forma sincónica o asincrónica para este primer acercamiento. $2^{\circ}$ Llamada. Este acercamiento debe realizarse de forma preferentemente sincrónica. El objetivo es observar a la díada representante-niño y que ellos puedan ver al profesional. Observar su relación , optimizar el diagnóstico, indicar el tratamiento, descender el riesgo familiar, indicar cuidados bucales familiares.

Se pueden realizar acciones de educación para la salud bucal en la familia, indicaciones para auto cuidado de adultos y niño con aplicación de Guías de Atención. En los países en los cuales las regulaciones lo permitan, se puede indicar tratamiento sintomático (analgésico, antibiótico).

$3^{\circ}$ Llamada. Retornos para teleconsulta con el objetivo de realizar seguimiento, controlar marcha del tratamiento y dar posible alta temporaria hasta finalización del confinamiento.

\section{Atención presencial:}

Solo cuando la anterior hubiese fracasado o fuera insuficiente para la solución del problema. La guía para la atención del niño que requiera atención presencial en tiempos de COVID-19 esta basada en Ruta de atención para procedimientos de Odontología Pediátrica durante la etapa de confinamiento o cuarentena de la pandemia COVID-19). ${ }^{19}$

La atención de urgencia a realizar en el momento de confinamiento debe estar orientada hacia mantener los dientes primarios funcionales y sanos en boca y realizar procedimientos que minimicen la generación de aerosoles. ${ }^{68,69}$

\section{Consulta presencial}

\section{Motivos de consulta presencial}

El tratamiento de las siguientes patologías se considera urgente y debe ser 
realizado incluso durante el periodo de confinamiento: pulpitis irreversible severa, pericoronaritis, absceso dentoalveolar localizado, fractura dental con dolor, alveolitis, trauma dental con avulsión o luxación, hemorragia, toma de biopsias o preparación odontológica previa a procedimientos médicos. ${ }^{4}$ En bebés, es necesario considerar la anquiloglosia y los dientes natales que dificultan la lactancia materna como urgencias neonatales. En bebés la atención debe ser prioritaria ya que en muchos casos no hay infecciones, dolor, trauma, absceso, pero hay lesiones de caries con una profundidad media que en 3 meses comúnmente pueden afectar la pulpa, incluso antes de que genere un dolor relevante para el bebé. El protocolo de la atención clínica debe seguir las pautas publicadas previamente por ALOP en el marco de la pandemia por COVID-19. ${ }^{71}$

\section{Dolor:}

Delimitar cuál es la magnitud del dolor es dificultoso en un niño pequeño.

Signos a tener en cuenta: llanto, frotar la cara en zona, enrojecimiento.

Ante la presencia de dolor leve o provocado que cesa, puede prescribirse Paracetamol (Acetaminofén) según peso del paciente y características del paciente y las directrices nacionales de salud de su país.

En caso de dolor más intenso o si el paciente ha tomado analgésicos por 48 horas sin mejoría, debe acudir a cita de urgencias.

\section{Traumatismos}

Avulsión de dientes primarios sin otra complicación asociada, fractura coronal simple de esmalte o de esmalte y dentina sin compromiso pulpar, o luxaciones leves sin interferencia oclusal ni movilidad, pueden ser tratados con telemedicina. En caso de luxaciones verificar magnitud del desplazamiento y si hay interferencia oclusal.

Tratamiento: Avulsión: control tejidos blandos no reimplantar. Luxaciones: indicar intentar realizar la reposición con presión digital (con excepción de luxaciones intrusivas).

\section{Pulpitis:}

La conducta terapéutica a seguir va orientada hacia lograr la detención de la lesión.

El manejo de la enfermedad caries en el marco de la Pandemia COVID-19 debe realizarse tomando como criterio la reducción de procedimientos que generen aerosoles.

Tratamiento: Aplicación de medidas preventivas a toda la familia. Uso técnica de Restauración Atraumática o remoción selectiva de caries. ${ }^{71}$

\section{Abscesos dento-alveolares}

Comenzar la resolución con Teleodontología durante la fase de pandemia aguda por COVID-19. Si no se logra, verificar dosis y medicación recetada. Medicación que puede ser indicada por Telemedicina. Amoxicilina, Amoxicilina + Ac. Clavulánico, Azitromicina, Clindamicina o el tratamiento farmacológico que decida el profesional de acuerdo a las regulaciones de su país.

La cita de urgencia puede planificarse 48 horas después del inicio de la toma de antibióticos de ser necesario. 


\section{Medidas se deben tomar en cuenta actualmente en el tratamiento odontológico del infante}

Las medidas que creemos esenciales son:

- Acompañamiento del padre/madre/ cuidador: durante la fase aguda y período posterior al pico de COVID-19. Es importante enfatizar a los padres que el niño deberá asistir con sólo un acompañante en la clínica dental, con el fin de evitar aglomeraciones y disminuir el riesgo de contagio. Asimismo, se le deberá recordar de no traer juguetes a la clínica. En el caso de traerlos, éstos deberán guardarse en una bolsa plástica antes de ingresar.

- Equipo de protección personal (EPP): una dificultad en la atención odontológica de bebés se relaciona con la limitación del niño pequeño para usar el EPP. Debido a que la presencia de un acompañante es inevitable, estas circunstancias podrían aumentar el riesgo de infección por COVID-19. Por ello, se vuelve imprescindible que, durante la atención del bebé, el acompañante del bebé use elementos del EPP, de acuerdo con las directrices de bioseguridad de cada país. Entre las barreras de protección para el acompañante se incluyen: mascarillas, batas / gorros descartables y gafas. Por otro lado, se sugiere que el bebé use gorro, babero descartable y gafas (si fuera posible).

\section{¿Cuáles técnicas alternativas en el manejo conductual durante el tratamiento odontológico del infante pueden usarse en tiempos de COVID 19?}

Independientemente de la edad del bebé, es necesario hacer un abordaje que reduzca los niveles de ansiedad y estrés de la triada.
Si hay necesidad de atención presencial del paciente, es importante tener un video o fotografías donde el Odontopediatra se vaya colocando todo el EPP y presente el nuevo ambiente odontológico y al equipo de trabajo. ALOP ha publicado material de apoyo audiovisual para orientación de los padres y profesionales. ${ }^{72}$

Otro recurso muy valioso es la música, que en el caso de neonatos que van a ser sometidos a cirugías de exodoncia de diente natal o para anquiloglosia, los esfuerzos estarán dirigidos a calmar a la madre. Siempre dar preferencias a músicas tranquilas que disminuyan la tensión del momento preferentemente seleccionadas por la madre. Es importante que en un contacto telefónico o virtual previo a la consulta presencial, la madre sea calmada y se le explique sobre toda la dinámica de la cirugía.

La música también puede ser usada para bebés y niños mayores de 3 años que requieren intervenciones dentales con el objetivo de reducir cualquier angustia generada con el procedimiento. ${ }^{73,74}$ Considerando que puede haber mayor cansancio para respirar del odontólogo con el uso de las máscaras y pantalla facial de forma continua y que además durante la pandemia se debe reducir el contacto y exceso de conversación en el ambiente clínico para reducir la contaminación, se sugiere que la música sea pasiva, es decir grabada con el uso de algún dispositivo de audio.

Es necesario que comprender la importancia del lenguaje no-verbal, principalmente durante estos tempos de pandemia, pues por medio de éste, el professional tendrá grandes oportunidades de ayudar al bebé, 
observando y percibiendo lo que sus gestos, su postura y su mirada quieren decir. ${ }^{67}$ De la misma forma, el bebé podrá sentir el deseo de cuidarlo y darle soporte emocional.

Por otro lado, los videos, y juegos interactivos para reducir la ansiedad preoperatoria de los niños parecen también ser efectivos. ${ }^{75,76}$ No se sugiere usar aromoterapia porque se desconoce el nivel de reacción con el virus.

\section{Consideraciones de salud bucal en casa}

Actualmente, la circunstancia de pandemia mundial que vivimos ha resaltado la importancia de la prevención de enfermedades bucales, especialmente de la caries dental. Los conceptos de cuidados en casa, educación a la familia, guías anticipatorias, hogar dental nunca han sido tan relevantes. Las consultas de mantenimiento y prevención podrían realizarse a través de Teleodontología con los padres, con el fin de promover comportamientos positivos de salud bucal.

\section{Consideraciones finales}

Estas recomendaciones han sido realizadas de forma independiente y voluntaria por los investigadores de la Asociación Latinoamericana de Odontopediatría, en conjunto con especialistas en las diversas áreas médicas en Latinoamérica, con arbitraje de pares, libres de conflicto de interés.

Las presentes recomendaciones están basadas en la mayor evidencia disponible hasta la fecha siendo el COVID-19 una enfermedad emergente. Se realizarán las actualizaciones necesarias conforme al estado de la ciencia al respecto y a la evolución de la enfermedad.

El grupo de expertos responsables de la curaduría se mantiene activo y en constante monitoreo de la información publicada referente a COVID-19 para realizar las actualizaciones y anexos necesarios.

Tabla 2.

\section{Recomendaciones sobre el cepillado dental}

Se debe usar una pasta dental con al menos 1.000 ppm de flúor en el cepillado dental. Éste debe hacerse al menos 2 veces al día ${ }^{77}$ desde la erupción del primer diente. La cantidad de dentífrico usada no debe ser superior a "un grano de arroz" hasta los 3 años ${ }^{78,79}$ y como una "arvejita" a partir de esta edad. Es de especial importancia levantar el labio superior al realizar el cepillado dental en los bebés, con el fin de cepillar correctamente los incisivos superiores.

\section{Recomendaciones sobre el consumo de azúcares libres}

Se debe evitar toda fuente de azúcares libres hasta los 2 años y posteriormente, se recomendará una dieta lo más baja posible en azúcares.

Se debe mantener una rutina alimentaria infantil adecuada en relación con la frecuencia de alimentos ofrecidos por día. Asimismo, se deben ofrecer alimentos in natura y mínimamente procesados.

Se debe incentivar el consumo de agua, evitando el consumo de jugos, refrescos y gaseosas, con el fin de evitar caries y desgaste dental erosivo. 


\section{Autores}

Desarrollo de este artículo liderado por: Jenny Abanto (Brasil), Haydée Casaretto González (Argentina), Bertha Angélica Chávez (Perú), Mónica Gladys Cesetti (Argentina), María Débora Elizabeth Dricas (Argentina), Sandra Echevarria (Brasil), Camila Palma (Perú), Gladys Mabel Peña (Argentina), Gabriel Politano (Brasil), como parte del Equipo Interdisciplinario COVID-19 de la Asociación Latinoamericana de Odontopediatría.

Equipo Interdisciplinario COVID-19 de la Asociación Latinoamericana de Odontopediatría:Jenny Abanto (Brasil), Mariana Armada (Argentina), Paola Beltri (España), Marisol Carrillo Tabakman (Paraguay), Haydée Casaretto (Argentina), Jorge Luis Castillo (Peru), Mónica Gladys Cesetti (Argentina), Bertha Angélica Chávez González (Peru), Ana Claudia Rodrigues Chibinski (Brasil), Salomón Alberto Cohen (Argentina), Olga Cortés Lillo (España), Luzia Ana Da Silva de Carballo (Venezuela), Gonzalo De la Fuente Alvarez (Chile), Renée Di Nallo (Argentina), María Débora Elizabeth Dricas (Argentina), Sandra Echevarria (Brasil), Piedad Cecilia Echeverry Marin (Colombia), Laura Fedelli (Argentina), Carlos FloresMIr (Canadá), Andrea Virgina González Carfora (Chile), Lina María Hernández Salas (Colombia),
Francisco José Hernández Restrepo (Colombia), Maria Teresa Ibañez Rodriguez (Bolivia), José Carlos Pettorossi Imparato (Brasil), Alejandra Lipari Valdés (Chile), Daniela Madrigal López (Costa Rica), Daniela Catalina Martínez Camus (Chile), María Gabriela Martínez Vásquez (Venezuela), A. Carolina Medina Díaz (Venezuela), Kelly Maria Silva Moreira (Brasil), Elías M. Morón (Estados Unidos de América), Martha Mutis (Estados Unidos de América), Camila Palma (Peru), Gladys Mabel Peña (Argentina), Adriana Pistochini (Argentina), Paloma Planells (España), Gabriel Politano (Brasil), Matias Rios-Erazo (Chile), Adriana Maria Rubiano Pinzon (Colombia), Karla Mayra Rezende (Brasil), Rosa Gabriela Rondón (Venezuela), Gabriela Scagnet (Argentina), Rosemary Sogbe de Agell (España), Marina Tavares Costa Nóbrega (Canadá), Jorgelina Valente (Argentina), Ernesto Venegas De Herrera (Republica Dominicana), Rosa Helena Wanderley Lacerda (Brasil), Ana Clara Zabala (Argentina), Gabriel Zambrano (Venezuela)

Información completa del Grupo Interdisciplinario COVID-19 de la Asociación Latinoamericana de Odontopediatría

\section{Referencias bibliográficas}

1. Boggess KA, Edelstein BL. Oral health in women during preconception and pregnancy: implications for birth outcomes and infant oral health.Matern Child Health J. 2006;10(5 Suppl):S169-74

2. Teshome A, Yitayeh A. Relationshipbetween periodontal disease and preterm low birthweight: systematic review. Pan Afr Med J. 2016;24:215.

3. Iheozor-Ejiofor Z, Middleton P, Esposito M, et al. Treating periodontal disease for preventing adverse birth outcomes in pregnant women. Cochrane DatabaseSyst Rev. 2017;6(6).

4. Musskopf ML, Milanesi FC, Rocha JMD, et al. Oral health related quality of life among pregnant women: a randomized controlledtrial. Braz Oral Res. 2018; 32:e02.

5. Fatturi AL, Wambier LM, Chibinski AC, et al. A systematic review and meta-analysis of systemic exposure associated with molar incisor hypomineralization. Community Dent Oral Epidemiol. 2019;47(5):407-415.

6. Huang C, Wang Y, Li X, et al. Clinical features of patients infected with 2019 novel coronavirus in Wuhan, China. Lancet. 2020; 15;395(10223):497-506.

7. Chen N, Zhou M, Dong X, et al. Epidemiological and clinical characteristics of 99 cases of 2019 novel coronavirus pneumonia in Wuhan, China: a descriptive study. Lancet 2020. 395:507e13.

8. Rasmussen SA, Smulian JC, Lednicky JA, Wen TS, Jamieson DJ, Coronavirus Disease 2019 (COVID-19) and Pregnancy: What obstetricians need to know, American Journal of Obstetrics and Gynecology (2020), doi: https://doi.org/10.1016/j.ajog.2020.02.017

9. Chen H, Guo J, Wang C, et al. Clinical characteristics and intrauterine vertical transmission potential of COVID-19 infection in nine pregnant women: a retrospective review of medical records. Lancet. 2020 Mar 7;395(10226):809-815. 
10. Zhu H,Wang L, Fang C, et al. Clinical analysis of 10 neonates born to mothers with 2019-nCoV pneumonia. Transl Pediatr 2020;9:51e60.

11. Centers for Disease Control and Prevention CDC. Interim Infection Prevention and Control Recommendations for Patients With Suspected or Confirmed Coronavirus Disease 2019 (Covid-19) in Healthcare Settings. abril 2020. Disponible en: https://www.cdc.gov/coronavirus/2019-ncov/ hcp/infection-control-recommendations.html?CDC_AA_refVal=https\%3A\%2F\%2Fwww.cdc. gov\%2Fcoronavirus\%2F2019-ncov\%2Finfection-control\%2Fcontrol-recommendations.html

12. Dave M, Seoudi N, Coulthard P. Urgent dental care for patients during the COVID-19 pandemic. The Lancet. Abril 2020:DOI: https://doi.org/10.1016/S0140-6736(20)30806-0

13. Ather A, Patel B, Ruparel NB, et al. Coronavirus Disease 19 (COVID-19): Implications for Clinical Dental Care. J Endod. 2020;46(5):584-595.

14. Equipo de trabajo multidisciplinario de la Revista de Odontopediatría Latinoamericana. Teleodontología: Aplicación a la Odontopediatría durante la pandemia COVID-19. Revista de Odontopediatría Latinoamericana. 2020; 10, (2), Obtenible en: https://www.revistaodontopediatria.org/ediciones/2020/2/ art-3/

15. ADA, 14 April 2020. Guidelines for Teledentistry https://www.ada.org.au/Covid-19 -Portal/Cards/ Dental-Profesionals/Practice-Policies/ADA-Guidelines-for-Teledentistry

16. Jampani ND, Nutalapati R, Dontula BS, et al. Applications of teledentistry: A literature review and update. J. Int. Soc. Prev. Community Dent. 2011;1(2):37-44.

17. Castaño de Casaretto H, Dricas D, Mayocchi K, et al. Circuitos de infectividad bucal entre madre-hijo. Estudio sobre información materna y disposición al cambio. Rev. Asoc. Odontol. Argent. (2003);91(4):305310.

18. ATDA (American Association OF Teledentistry.Teledental Practice and Teledental) Encounters: an American Association of Teledentistry Position Paper. 2018. https://www.americanteledentistry.org/ wpcontent/uploads/2018/10/ATDA_TeledentalPracticePositionPaper.pdf

19. Asociación Latinoamericana de Odontopediatría. Grupo COVID-19. Ruta de atención para procedimientos de Odontología Pediátrica durante la etapa de confinamiento o cuarentena de la pandemia COVID-19. Revista de Odontopediatría Latinoamericana. 2020; 10(2): https://www.revistaodontopediatria.org/ ediciones/2020/2/art-1/.

20. Jin Xiao, NaemahAlkhers, Dorota $\mathrm{T}$ Kopycka-Kedzierawski, et al. Prenatal Oral Health CareandEarlyChildhood Caries Prevention: A Systematic Reviewand Meta-Analysis. Caries Res. 2019; 53(4):411-421.

21. Riggs E, Kilpatrick N, Slack-Smith L, et al. Interventions with pregnant women, new mothers and other primary caregivers for preventing early childhood caries. Cochrane Database Syst Rev. 2019;2019(11):CD012155.

22. Williams J, Namazova-Baranova L, Weber M, et al. The importance of continuing Breastfeeding during COVID-19: in support to the WHO Statement on Breastfeeding during the pandemic. J Pediatr 2020;10; S0022-3476(20)30583-7.

23. Lackey KA, Pace RM, Williams JE, et al. SARS-CoV-2 and human milk: What is the evidence? Matern Child Nutr 2020; 30:e13032.

24. Duran P, Berman S, Niermeyer S, et al. COVID-19 and newborn health: systematic review. Rev Panam Salud Publica 2020; 44:e54.

25. Giuliani C, Li Volsi P, Brun E, et al. Breastfeeding during the COVID-19 pandemic: suggestions on behalf of Woman Study Group of AMD. Diabetes Res Clin Pract 2020; 29:108239.

26. Kimberlin DW, Stagno S. Can SARS-CoV-2 infection be acquired in utero? More definitive evidence is needed. JAMA. 2020 Mar 26. doi: 10.1001/jama.2020.4868. Online ahead of print.

27. Karimi-Zarchi M, Neamatzadeh H, Dastgheib SA, et al. Vertical transmission of Coronavirus Disease 19 (COVID-19) from infected pregnant mothers to neonates: a review. Fetal Pediatr Pathol. 2020;39(3):246-250.

28. Walker KF, O-Donoghue K, Grace N, et al. Maternal transmission of SARS-COV-2 to the neonate, and possible routes for such transmission: a systematic review and critical analysis. BJOG. 2020 Jun 12. doi: 10.1111/1471-0528.16362. Online ahead of print.

29. Centers for Disease Control and Prevention (CDC). Coronavirus disease 2019 (COVID-19). Care for Breastfeeding Women: Interim Guidance on Breastfeeding and Breast milk feeds in the context of Covid-19. 9 de Junio 2020. Disponible en: https://www.cdc.gov/coronavirus/2019-ncov/hcp/care-forbreastfeeding-women.html [Accedido 13 de Junio, 2020]. 
30. OMS: Organización Mundial de la Salud (2020). Clinical management of COVID-19. 27 de Mayo 2020, Ginebra. Disponible en: https://www.who.int/publications/i/item/clinical-management-of-covid-19

31. World Health Organization infographics: Pregnancy, Childbirth, breastfeeding and COVID-19. Accesible en: $\quad$ https://www.who.int/reproductivehealth/publications/emergencies/COVID-19-pregnancy-ipcbreastfeeding-infographics/en/ [13 de Junio, 2020]

32. OMS, Preguntas frecuentes: lactancia materna y COVID-19 para trabajadores de la salud, 12 de Mayo 2020. Disponible en: https://www.who.int/docs/default-source/coronaviruse/breastfeeding-covid-whofaqs-es-12may2020.pdf?sfvrsn=f1fdf92c_8 [Accedido 14 de Junio 2020]

33. European Pediatric Association (EPA)- Union of National European Pediatric Societies and Associations (EPAUNESPA). Disponible en: http://www.epa-unepsa.org./ [Accedido 13 de Junio, 2020]

34. UNICEF: Breastfeeding during the COVID-19 pandemic. Disponible en: https://www.unicef.org/eap/ breastfeeding-during-covid-19 [Accedido 13 de Junio 2020]

35. ISUOG: International Society in Ultrasound for Obstetrics and Gynecology; New Interim Guidance on 2019 coronavirus infection during pregnancy and puerperium: information for healthcare professionals. Disponible en: https://www.isuog.org/resource/new-isuog-interim-guidance-2019-novel-coronavirusinfection-during-pregnancy-and-puerperium-information-for-healthcare-professionals.html [Accedido 13 de Junio, 2020]

36. RCOG: Royal College of Obstetricians and Gynaecologists. Coronavirus (COVID-19) Infection in pregnancy: information for healthcare professionals. Updated 4th of June, 2020. Disponible en: https:// www.rcog.org.uk/coronavirus-pregnancy [Accedido 13 de Junio, 2020]

37. ABM: Academy of Breastfeeding Medicine. ABM Statement on Coronavirus 2019 (COVID-19), March 10th, 2020. Disponible en: https://www.bfmed.org/abm-statement-coronavirus [Accedido 13 de Junio, 2020]

38. Fernández-Carrasco FJ, Vásquez-Lara JM, González-Mey U, et al. [Coronavirus Covid-19 infection and breastfeeding: an exploratory review]. Rev Esp Salud Publica 2020 May 27; 94:e202005055.

39. ADA: American Dental Association: What constitutes a dental emergency? (March 31st, 2020). Disponible en: https://success.ada.org/ /media/CPS/Files/Open\%20Files/ADA_COVID19_Dental_Emergency_DDS. pdf [Accedido 14 de Junio 2020]

40. EsSalud, Perú: recomendaciones clínicas para realizar procedimientos en Odontoestomatología en el contexto de pandemia por Covid-19. Disponible en: http://www.essalud.gob.pe/ietsi/pdfs/guias/ Recomendaciones_OdontoestomatologIa_COVID.pdf [Accedido 14 de Junio, 2020]

41. Colegio de Odontólogos y Estomatólogos de Catalunya: Recomendaciones e infografías para la atención de urgencias dentales durante la pandemia (30 de Marzo, 2020). Disponible en: http://www.coec.cat/es/ noticies/atencio-urg-dentals-covid19 [Accedido 14 de Junio 2020]

42. SDCEP: Scottish Dental Clinical Effectiveness Programme: Management of acute dental problems during COVID-19 Pandemic, March 2020. Disponible en http://www.sdcep.org.uk/published-guidance/acutedental-problems-covid-19/ [Accedido 14 de Junio 2020]

43. Consejo General de Colegio de Dentistas de España: Plan estratégico de acción para el periodo posterior a la crisis creada por el COVID-19, Abril 2020. Disponible en: https:/www.consejodentistas.es/ comunicacion/actualidad-consejo/notas-de-prensa-consejo/item/1763-plan-estrategico-de-accion-parael-periodo-posterior-a-la-crisis-creada-por-el-covid-19.html [Accedido 14 de Junio 2020]

44. Costacurta M, Maturo P, Docimo R. Riga-Fede disease and neonatal teeth. Oral Implantol (Rome). 2012;5(1):26-30.

45. Bulut G, Bulut H, Ortac R. A comprehensive survey of natal and neonatal teeth in newborns. Niger J Clin Pract. 2019;22(11):1489-1494.

46. Meng L, Hua F, Bian Z. Coronavirus Disease 2019 (COVID19): Emerging and Future Challenges for Dental and Oral Medicine. J Dent Res. 2020 May;99(5):481-487.

47. Organización Mundial de la Salud (OMS). Desarrollo de la primera infancia: un potente ecualizador. 2007. Disponible en: https://www.who.int/social_determinants/publications/early_child_dev_ecdkn_ es.pdf?ua $=1$

48. Chang TH, Wu JL, Chang LY. Clinical characteristics and diagnostic challenges of pediatric COVID-19: A systematic review and meta-analysis. J Formos Med Assoc. 2020; 119(5): 982-989.

49. Liguoro I, Pilotto C, Bonanni M, et al. SARS-COV-2 infection in children and newborns: a systematic review. Eur J Pediatr. 2020;1-18. 
50. Mustafa NM, A Selim L. Characterisation of COVID-19 Pandemic in Paediatric Age Group: A Systematic Review and Meta-Analysis J Clin Virol. 2020;128:104395.

51. Castagnoli R, Votto M, Licari A, et al. Severe Acute Respiratory Syndrome Coronavirus 2 (SARS-CoV-2) Infection in Children and Adolescents: A Systematic Review JAMA Pediatr. Published online April 22, 2020. doi:10.1001/jamapediatrics.2020.1467.

52. Departamentos Científicos de Adolescência (2019-2021) e de Pediatria do Desenvolvimento e Comportamento (2019-2021). Ouso de máscaras faciais em tempo de COVID-19 por crianças eadolescentes: Uma proposta inicial. Disponible en: https://www.sbp.com.br/fileadmin/user_upload/22543e-NA_-_O_ uso_mascaras_faciais_em_COVID19_por_crc_e_adl_1_.pdf

53. Academia Americana de Pediatria - HEALTHY CHILDREN. 2019 Novel Coronavirus (COVID-19). 2020. Disponível em: https://www.healthychildren.org/English/health-issues/conditions/chest-lungs/ Pages/2019-Novel-Coronavirus.asp Acessado em 13/05/2020.

54. Centers for Disease Control and Prevention CDC. Interim Infection Prevention and Control Recommendations for Patients With Suspected or Confirmed Coronavirus Disease 2019 (Covid-19) in Healthcare Settings. abril 2020. Disponible en: https://www.cdc.gov/coronavirus/2019-ncov/ hcp/infection-control-recommendations.html?CDC_AA_refVal=https\%3A\%2F\%2Fwww.cdc. gov\%2Fcoronavirus\%2F2019-ncov\%2Finfection-control\%2Fcontrol-recommendations.html.

55. Comina E, Marion K, Renaud FN, Dore J, Bergeron E, Freney J. Pacifiers: amicrobial reservoir. Nurs Health Sci 2006; 8(4):216-23.

56. Mattos Graner RO, de Moraes AB, Rontani RM, et al. Relation of oral yeast infection in Brazilian infants and use of a pacifier. ASDC J Dent Child 2001; 68(1):33-6.

57. Hesselmar B, Sjöberg F, Saalman R, et al. Pacifier cleaning practices and risk of allergy development. Pediatrics. 2013;131(6):e1829-37.

58. North K, Fleming P, Golding J, and the ALSPAC study team. Pacifier use and morbidity in the first six months of life. Pediatrics.1999;103(3).

59. Souza VGC, Lopes DF, Machado FC, et al. The Novel Coronavirus: An Alert for Pacifiers' Disinfection. Pesqui Bras Em Odontopediatria e Clínica Integrada 2020; 20, e0071.

60. World Health Organization Pandemic Phase Descriptions. https:// www.who.int/influenza/resources/ documents/pandemic_phase_descriptions_and_actions.pdf. Accesado en 13 Junio, 2020.

61. Mallineni SK, Innes NP, Raggio DP, et al. Coronavirus disease (COVID-19): Characteristics in children and considerations for dentists providing their care. Int J Paediatr Dent. 2020;30:245-250.

62. Conselho Federal de Odontologia (CFO). Exercício da Odontologia a distância, mediado por tecnologias, e dá outras providências. RESOLUÇÃO CFO-226. En: http://www.crosp.org.br/uploads/arquivo/ e1eb52fada70ddc938113152ba2b9cea.pdf. Accesado en 13 Junio, 2020.

63. Souza RCC, Costa OS, Costa LR. Dental sedation precautions and recommendations during the COVID-19 Pandemic. Rev Bras Odontol. 2020;77:e1788.

64. Franco JB, Camargo AR, Peres MPSM. Cuidados Odontológicos na era do COVID-19: recomendações para procedimentos odontológicos e profissionais. Rev Assoc Paul Cir Dent.2020;74(1):18-21.

65. Prescilla RP, Mason KP. Sedation and General Anesthesia. En: Treves ST. Pediatric Nuclear Medicine and Molecular Imaging. New York: Springer. 2014.

66. Souza RCC. Odontologia especial pediátrica: correlação prática e evidências. São Paulo: Quintessence Editora. 2019.

67. Abanto J, Konishi F, Noronha JC. Paso a paso para el abordaje del comportamiento no farmacológico en el paciente odontopediátrico. En: Abanto J, Rédua PCB, Bönecker M. Paso a paso para conductas clínicas en Odontopediatría. São Paulo: Santos Publicações, 2020. pp.19-45.

68. Centers for Disease Control and Prevention CDC. Recommendation: Postpone Non-Urgent Dental Procedures, Surgeries, and Visits. Marzo 27, 2020. Disponible en: https://www.cdc.gov/oralhealth/ infectioncontrol/statement-COVID.html.

69. American Academy of Pediatric Dentistry. COVID-19 Update/Coronavirus Update. 2020 Abril. Disponible en: https://www.aapd.org/about/about-aapd/news-room/covid-19/.

70. Dave M, Seoudi N, Coulthard P. Urgent dental care for patients during the COVID-19 pandemic. The Lancet. Abril 2020:DOI: https://doi.org/10.1016/S0140-6736(20)30806-0.

71. Asociación Latinoamericana de Odontopediatría (ALOP). Tratamiento de caries en época de COVID-19: Protocolos clínicos para el control de generación de aerosoles. Revista de Odontopediatría Latinoamericana, 10(2).2020. 
72. Equipo de trabajo multidisciplinario de la Revista de Odontopediatría Latinoamericana. Estrategias Psicoconductuales e información para Odontólogos y pacientes que requieran procedimientos de Odontología Pediátrica durante la etapa de la pandemia COVID-19. Revista de Odontopediatría Latinoamericana. 2020; 10 (2). Obtenible en: https://www.revistaodontopediatria.org/ediciones/2020/2/ art-5/

73. Franzoi MA, Goulart CB, Lara EO, et al. Music listening for anxiety relief in children in the preoperative period: a randomized clinical trial. Rev Lat Am Enfermagem. 2016;24:e2841.

74. Van der Heijden MJE, Mevius H, van der Heijde N, van Rosmalen J, et al. Children Listening to Music or Watching Cartoons During ER Procedures: A RCT. J Pediatr Psychol. 2019;44(10):1151-1162.

75. Barreiros D, de Oliveira DSB, de Queiroz AM, et al. Audiovisual distraction methods for anxiety in children during dental treatment: A systematic review and meta-analysis. J Indian Soc Pedod Prev Dent. 2018;36(1):2-8.

76. Chow CH, Van Lieshout RJ, Schmidt LA, et al. Systematic Review: Audiovisual Interventions for Reducing Preoperative Anxiety in Children Undergoing Elective Surgery. J Pediatr Psychol. 2016;41(2):182-203.

77. Walsh T, Worthington HV, Glenny AM, Marinho VC, Jeroncic A. Fluoride toothpastes of different concentrations for preventing dental caries. Cochrane Database Syst Rev 2019;3:Cd007868.

78. Wright JT, Hanson N, Ristic H, Whall CW, Estrich CG, Zentz RR. Fluoride toothpaste efficacy and safety in children younger than 6 years: a systematic review. J Am Dent Assoc 2014;145:182-9.

79. Chedid SJ, Tenuta LM, Cury JA. Amount of F-dentifrice used by young children and saliva/biofilm fluoride. J Dent Res. 2011;90:Abstract 2637.

Recibido: 10/06/2020

Aceptado: 31/07/2020

Correspondencia: e-mail: editor@revistaodontopediatria.org 
Anexo 1

\section{ALQ̈P ASOCIACIÓN LATINOAMERICANA DE ODONTOPEDIATRÍA}

\section{RUTA DE ATENCIÓN PARA PROCEDIMIENTOS ODONTOLÓGICOS EN LA GESTANTE DURANTE CONFINAMIENTO O CUARENTENA POR LA PANDEMIA POR COVID-19}

\section{Consulta de Urgencia-Emergencia}

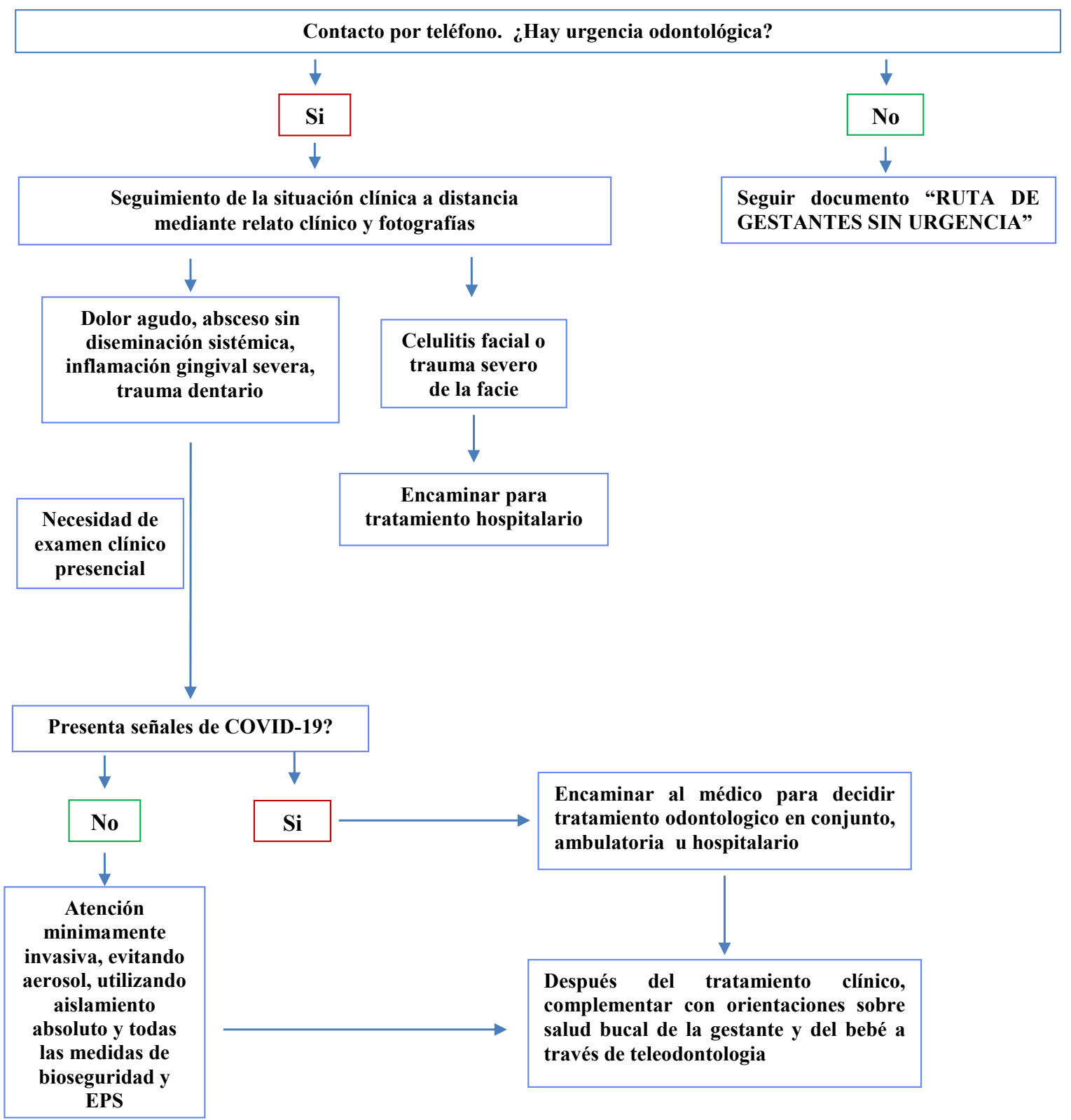


Anexo 2

\section{ALOPASOCIACIÓN LATINOAMERICANA DE ODONTOPEDIATRÍA}

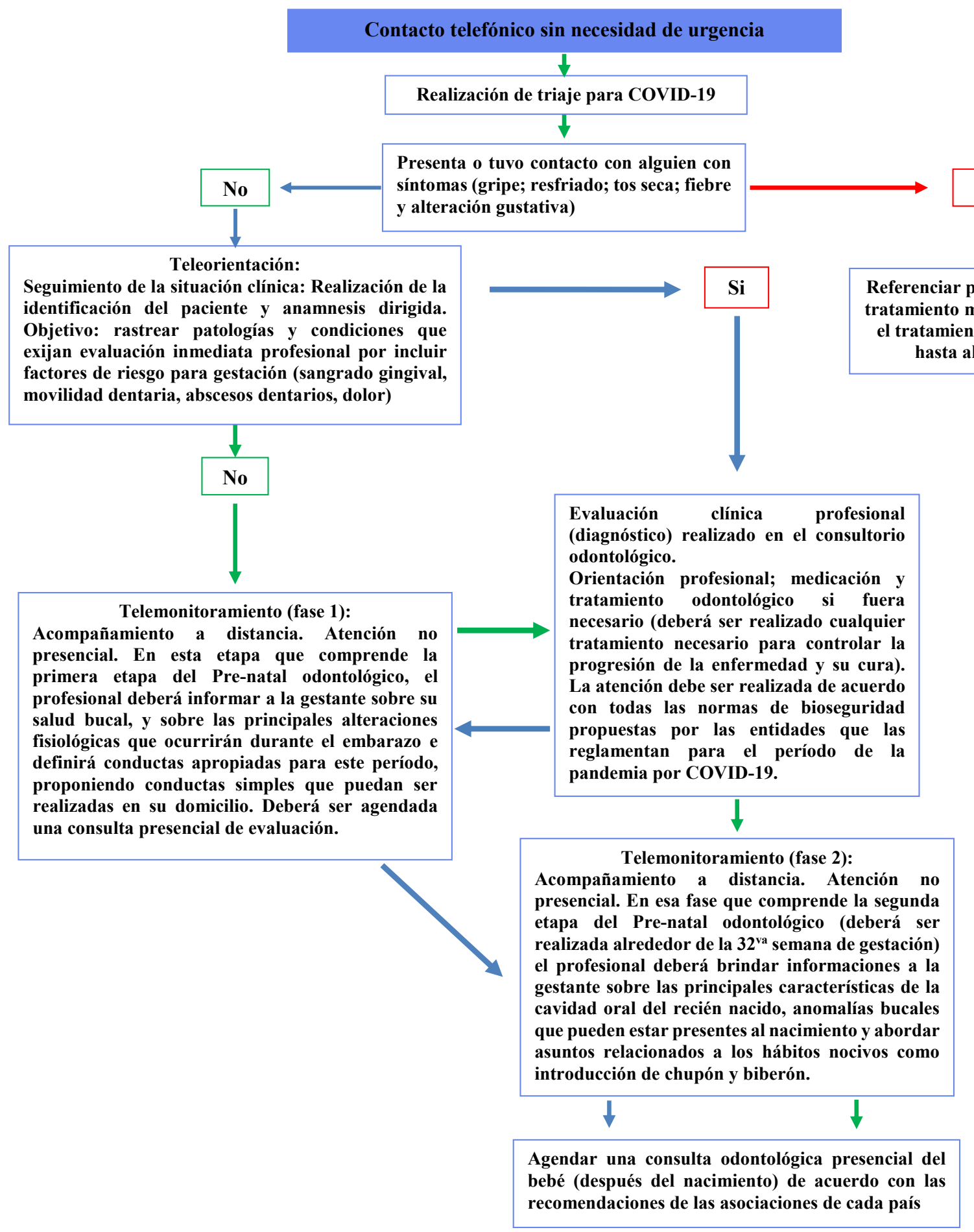

Todo telemonitoramiento realizado con la gestante deberá ser registrado en la historia clínica de la paciente y la teleorientación debe tener como objetivo único y exclusivo identificar el mejor momento para la atención presencial 